\section{A Atenção Primária à Saúde \\ no sul de Santa Catarina: uma \\ análise das internações por \\ condições sensíveis à atenção \\ ambulatorial, no período de \\ 1999 a 2004}

\section{Primary Health Care in the south \\ of Santa Catarina: analysis of \\ admissions for ambulatory \\ care-sensitive conditions, in the \\ 1999-2004 period}

\section{Evelyn Elias ${ }^{1}$}

\section{Flávio Magajewski ${ }^{2}$}

${ }^{1}$ Programa de Mestrado em Ciências da Saúde da Universidade do Sul de Santa Catarina-UNISUL , Docente do Curso de Enfermagem da Universidade do Sul de Santa Catarina-UNISUL

${ }^{2}$ Programa de Mestrado em Ciências da Saúde da Universidade do Sul de Santa Catarina-UNISUL, Docente do Curso de Medicina da Universidade do Sul de Santa Catarina-UNISUL

Correspondência: Evelyn Elias, Avenida Sete de Setembro, 2030, Araranguá, SC, CEP 88900-000 E-mail: evelyn.elias@unisul.br

\section{Resumo}

Introdução: A reorientação do modelo de atenção à saúde brasileira tem como principal estratégia a estruturação de uma rede de atenção primária que dê cobertura às necessidades de saúde da população através do acesso a equipes de saúde da família. A avaliação do impacto da atenção primária sobre a saúde da população pode ser feito a partir do monitoramento das internações hospitalares por condições sensíveis à atenção ambulatorial, iniciativa que vem se desenvolvendo nos últimos anos em vários países e também no Brasil. Objetivo: analisar o comportamento das internações hospitalares por algumas condições sensíveis à atenção ambulatorial na região da Associação de Municípios do Extremo Sul de Santa Catarina - AMESC, associando-o com a qualidade da atenção oferecida pelo Programa de Saúde da Família (PSF). Método: Pesquisa exploratória com características de estudo ecológico que qualificou a atenção primária oferecida a partir de questionário dirigido aos profissionais do PSF e informações colhidas através dos bancos nacionais de informação, definindo dois grupos de população: uma atendida por serviços de atenção básica com padrão adequado e outra servida por serviços abaixo desse padrão. As internações hospitalares por algumas causas selecionadas destas duas populações foram comparadas no período 1999-2004, analisando-se a sua tendência e associação estatística. Resultados: A análise estatística sugeriu que houve tendência ao declínio das taxas de internações por pneumonia em menores de 5 anos e pneumonia em maiores de 60 anos na população com atenção ambulatorial básica adequada e não adequada. As internações por Diabetes Mellitus tiveram tendência ao declínio na população com atenção ambulatorial básica adequada. As taxas por diarréia apresentaram tendência à estabilidade nos dois grupos populacionais, mas nos municípios com atenção adequada a sua magnitude foi muito menor. As taxas de internação por Acidente Vascular Cerebral foram crescentes em município 
com atenção adequada, com tênue queda linear para a população atendida de forma não adequada. Em relação às internações por Infarto Agudo do Miocárdio, tanto nos municípios com atenção adequada quanto naqueles com atenção não adequada, as taxas apresentaram tendência ao aumento, com tendência um pouco menor nos municípios com PSF adequado. Conclusão: É possível perceber algumas mudanças positivas na evolução de algumas taxas de internações por condições sensíveis à atenção ambulatorial, que podem estar associadas a maior cobertura e a melhor atenção recebida pelo PSF.

Palavras-chave: Programa de Saúde da Família. Qualidade da atenção. Avaliação em saúde. Internações por causas sensíveis à atenção ambulatorial.

\section{Abstract}

Introduction: The reorientation of the health care model in Brazil is based on a strategy to structure a primary care network to cover the health needs of the population through access to family health teams. The impact of primary care on the health of the population can be assessed by tracking hospital admissions due to conditions sensitive to ambulatory care, an initiative that has been developed in recent years in several countries and also in Brazil. Objective: To analyze hospital admission trends for some conditions sensitive to ambulatory care in the Association of Cities of the South End of Santa Catarina - AMESC, associating it to the quality of care offered by the Family Health Program-PSF. Method: An exploratory research with characteristics of an ecological study that qualified the primary care offered, based on a questionnaire directed to PSF professionals and information collected through national data information, defining two population groups: one assisted by services with a basic standard and another one assisted by services below this standard. Hospital admissions for some selected causes of these two populations were compared in the 1999-2004 period, analyzing their trend and statistical association. Results: The statistical analysis suggested that there was a trend toward the decline of admissions due to pneumonia in $<5 \mathrm{y}$-o and $>60 \mathrm{y}$-o in both the population with appropriate basic and non-appropriate ambulatory care. Diabetes mellitus hospitalizations tended to decline in the population with adjusted basic ambulatory care. Diarrhea rates tended to stabilize in both groups, but in the cities with appropriate care its magnitude was much smaller. Strokes increased in cities with appropriate care, with a smooth linear decrease for the population assisted in a non-appropriate way. For Acute Myocardial Infarction, for cities with appropriate care, as for the ones with non-appropriate care, the rates showed a trend toward increase, although a little smaller in the cities with appropriate PSF. 
Conclusion: Some positive changes can be perceived in the development of admission rates due to ambulatory care-sensitive conditions, that can be associated with the higher coverage and the best care received from the PSF.

Keywords: Family Health Program. Quality of care. Healthcare evaluation. Ambulatory care-sensitive admissions.

\section{Introdução}

O Programa Saúde da Família-PSF surgiu em 1994 e gradativamente vem se consolidando como uma estratégia bem sucedida de reorganização do nível de atenção básica do sistema de saúde brasileiro, fundamentada nos princípios da Atenção Primária à Saúde (APS), cujo desafio é concretizar a atenção integral, continuada e resolutiva à saúde da população brasileira ${ }^{1-10}$.

Bons resultados têm sido obtidos em diversos países onde os sistemas de saúde são orientados por estes princípios. A atenção primária está associada a custos menores, maior satisfação dos usuários e melhores indicadores de saúde, mesmo em situações de grande iniqüidade social ${ }^{5,11-18}$.

No Brasil, os resultados atribuíveis à parte do sistema de saúde fundamentada na atenção primária também têm sido positivos, ainda que freqüentemente contradições de natureza político-ideológica ou conceitual sejam apresentadas para relativizá- $\operatorname{los}^{3,5,14}$. Quando confrontamos a expansão acelerada da estratégia de Saúde da Família com o cotidiano relativamente restritivo das Equipes de Saúde da Família (ESF) em relação às suas ambiciosas responsabilidades, alguns questionamentos acerca da capacidade de transformação da atenção básica ainda não têm resposta segura: A atenção básica reestruturada dentro desta lógica está efetivamente exercendo o papel de porta de entrada do Sistema Único de Saúde? Os princípios que norteiam a atenção primária estão efetivamente se concretizando no cotidiano dos serviços? Os resultados positivos já mensurados estão relacionados apenas à ampliação de cobertura e acesso ou está ocorrendo uma mudança qualitativa nas relações entre os serviços de saúde e a sua clientela?

Para obter respostas a esses questionamentos, buscou-se estudar o PSF como estratégia estruturada em torno de responsabilidades, competências, atividades, objetivos, resultados e impactos esperados, articulando-se a um padrão assistencial 
desejado. Baseados na premissa de que o PSF tem como principais características a responsabilidade sanitária sobre um determinado território com uma população adscrita, realizamos um estudo focando as ESF implantadas na região sul de Santa Catarina, avaliando o seu funcionamento e a associação desta atuação com um determinado desfecho, as internações da população por algumas causas consideradas sensíveis à atuação da atenção primária de saúde. Para tal os serviços baseados na ESF foram avaliados e classificados em dois grupos (como ofertantes de atenção adequada ou não adequada segundo algumas variáveis e critérios) e comparados em relação ao comportamento das internações por condições sensíveis à atenção ambulatorial - ICSAAs, no período de 1999 a 2004.

Consideramos ICSAAs as hospitalizações que podem ser eventualmente reduzidas e/ ou evitadas se forem correta e efetivamente diagnosticadas e tratadas no nível ambulatorial, ou seja, se a atenção primária à saúde for resolutiva ${ }^{5,13}$. Nesse sentido, assumimos o percentual de ICSAAs como um indicador de qualidade da atenção à saúde. Em síntese, a magnitude das internações hospitalares por algumas causas selecionadas reflete a qualidade da atenção ou as linhas de cuidado oferecidas à população, avaliando elementos de estrutura e processo das unidades de saúde e equipes de saúde associadas com essa atenção.

Nos últimos anos, tem-se observado o empenho das estruturas políticas governamentais em adotar a avaliação como prática regular e sistemática de suas ações. Pesquisadores em diversos países vêm desenvolvendo estudos direcionados à construção de indicadores que possam avaliar a capacidade de resolução da Atenção Primária, sendo a análise das ICSAAs um dos indicadores propostos para avaliar resultado, efeito ou impacto da atenção oferecida neste nível do sistema de saúde $\mathrm{e}^{5,11,14-9}$.

O termo Internações por Condições Sensíveis à Atenção Ambulatorial (ICSAAs) originou-se nos Estados Unidos na década de noventa, com a denominação Ambula- tory Care Sensitive (condições sensíveis à atenção ambulatorial), também chamada Avoidable Hospitalization (hospitalizações evitáveis). Este indicador foi utilizado inicialmente para analisar a acessibilidade da população indigente aos serviços de saúde, mas na segunda metade da década de noventa foi proposto pelo National Health Service para a análise da qualidade da Atenção Primária e tem sido associado inversamente à cobertura de seguro-saúde e ao padrão socioeconômico da população assistida $^{5,14}$

$\mathrm{Na}$ Espanha ${ }^{18-20}$, as pesquisas têm procurado validar uma relação de causas de internações Hospitalares Sensíveis à Atenção Primária para medir a efetividade da Atenção Primária à Saúde, por entender que estas podem variar de acordo com os serviços de saúde oferecidos neste nível de atenção. Caminal et al. ${ }^{21}$ estudaram, em várias regiões da Espanha, 35 códigos de diagnósticos de internação hospitalar distribuídos em treze grupos de enfermidades, todos associados às CSAAs. Os resultados apontaram para associação das taxas de hospitalizações por CSAAs com as características da organização da atenção primária, o tamanho do município e a distância dos hospitais de referência.

Na região da Cataluña ${ }^{20,21}$, estudo com praticamente a mesma concepção, mostrou que entre 1998 e 1999, de um total de 1.376.632 internações hospitalares ocorridas, $8,42 \%$ (116.006) foram categorizadas como Internações Sensíveis à Atenção Primária. Deste Percentual, 86,9\% estavam relacionadas ao grupo de enfermidades cardiovascular e hipertensão arterial $(40,7 \%)$, de insuficiência cardíaca $(24,8 \%)$, de pneumonia (15\%) e de infecções do trato urinário $(6,4 \%)$.

No Brasil, o Ministério da Saúde ${ }^{1}$ indicou uma importante redução das internações infantis a partir da implantação do PSF, e cita como fatores que contribuíram para essa redução o acompanhamento sistemático e a garantia de acesso às crianças com problemas agudos ao atendimento médico nas unidades de saúde ${ }^{22-25}$. 
Estudo realizado em hospitalizações por causas evitáveis em crianças de um a quatro anos na Região Nordeste do Brasil, durante o ano de 1994, encontrou taxa de hospitalização de $24,4 \%$, sendo a maioria por pneumonia $(7,3 \%)$ e diarréia $(7,1 \%)$, e na sua maioria custeadas pelo Sistema Único de Saúde - SUS (78,1\%). $\mathrm{Na}$ avaliação dos autores, a maior parte das hospitalizações na região ocorreu por causas evitáveis ou sensíveis à atenção ambulatorial ${ }^{24}$.

Alfradique e Mendes ${ }^{9}$ realizaram estudo avaliando as internações do SUS no ano de 2001, demonstrando que, de um total de 12.426.111 internações pagas pelo SUS, 3.405.452 foram por condições sensíveis à atenção ambulatorial, ou $27,4 \%$ do total das internações desse ano. A retirada dos partos do universo pesquisado elevaria esse percentual para $33,8 \%$.

As taxas de hospitalização têm sido utilizadas de forma crescente como indicadores na avaliação de programas de atenção primária, especialmente em estudos com base populacional, buscando avaliar em que medida programas ou sistemas de APS, ao atingir suas metas, melhoram a condição de saúde da população assistida. Há evidências de que serviços de atenção primária de melhor qualidade estão associados a taxas mais baixas de internações por condições sensíveis à atenção ambulatorial (ICSAAs) $)^{5,13,25}$. Supõe-se que disparidades nas internações por certas doenças consideradas de fácil prevenção, ou por aquelas que seriam passíveis de diagnóstico e tratamento precoce de modo a evitar a hospitalização, refletem a inadequação da atenção à saúde às necessidades de determinadas comunidades.

Da mesma forma, essas informações, quando associadas aos dados de outros serviços que compõem o sistema de assistência à saúde, podem refletir as condições de vida e saúde da população e permitem a avaliação indireta da estrutura dos serviços de saúde, da assistência oferecida e da política médico-assistencial ${ }^{25}$.

\section{Métodos}

A pesquisa desenvolvida caracteriza-se como exploratória com característica de um estudo ecológico, associando a qualidade da atenção primária oferecida através do PSF à população de determinados municípios com as internações hospitalares por algumas condições sensíveis à atenção primária, no período de 1999 a 2004.

A região selecionada foi a Associação dos Municípios do Extremo Sul Catarinense (AMESC), com 15 municípios, cujo pólo regional está localizado no Município de Araranguá - SC. Com base nos dados do Instituto Brasileiro de Geografia e Estatística (IBGE-2000), a região possuía 160.078 habitantes, $34,17 \%$ dos quais residiam no município pólo. Nesta região, 73,33\% dos municípios têm população inferior a 10.000 habitantes ${ }^{26}$. Para o período de 2000 a 2004, a região obteve o conceito de médio baixo no Índice dos Objetivos do Desenvolvimento do Milênio-IODM ${ }^{27}$.

A rede hospitalar na região estudada era composta por sete unidades (dezembro de 2004), totalizando 380 leitos para o atendimento das quatro clínicas básicas, sendo que, destes, 280 eram credenciados pelo SUS. Apenas o hospital do município pólo possuía mais de cem leitos (105 leitos); outro possuía 77 leitos e os demais tinham características de hospitais de pequeno porte, com menos de 50 leitos $^{28}$.

Foram utilizadas como unidades de análise dois conjuntos de municípios agrupados segundo a cobertura do Programa Saúde da Família no ano de 2004 e a qualidade da atenção básica oferecida à população pelas suas Equipes de Saúde da Família - ESFs, conformando duas unidades ecológicas, ou seja, municípios com atenção básica adequada e atenção básica não adequada. Os critérios adotados para a inclusão na unidade ecológica adequada foram municípios com $70 \%$ ou mais de cobertura do PSF, de acordo com modelo analítico proposto por Mendes ${ }^{9}$, e pelo menos dois terços de suas ESFs qualificadas para a ação e serviços da Atenção Básica, segundo critérios estabelecidos pelos autores (Tabela 1). 
Tabela 1 - Categorias operativas, componentes de estrutura e processo, parâmetros e fontes das informações. Table 1 - Operative categories, structure and process components, parameters and information sources.

\begin{tabular}{|c|c|c|c|}
\hline Categorias Operativas & Componentes de estrutura e processo & Parâmetros adotados & Fonte das informações \\
\hline \multirow{7}{*}{ Primeiro Contato } & Mapeamento do território - área atualizada; & $\begin{array}{l}\text { Atualização nos últimos } \\
\text { três meses; }\end{array}$ & Questionário \\
\hline & $\begin{array}{l}\text { ESF com cadastramento da população de } \\
\text { área adscrita; }\end{array}$ & Até 4500 pessoas; & Questionário \\
\hline & $\begin{array}{l}\text { Horário de funcionamento da unidade de } \\
\text { saúde; }\end{array}$ & Mínimo de 8 horas/dia; & Questionário \\
\hline & $\begin{array}{l}\text { Acesso para portadores de deficiências físicas } \\
\text { e idosos; }\end{array}$ & 100\% de adequação; & Questionário \\
\hline & Estrutura física da USF adequada; & 90\% de adequação; & Questionário \\
\hline & Marcação de consulta & $\begin{array}{l}\text { Garantia de } 100 \% \text { às } \\
\text { áreas prioritárias; }\end{array}$ & Questionário \\
\hline & Turno de atendimento da ESF - horário & $\begin{array}{l}\text { Mínimo de } 2 \text { turnos com } \\
4 \text { horas cada; }\end{array}$ & Questionário \\
\hline \multirow{11}{*}{$\begin{array}{l}\text { Integralidade } \\
\text { (abrangência) }\end{array}$} & $\begin{array}{l}\text { Oferta de atividades educativas e } \\
\text { terapêuticas a grupos populacionais } \\
\text { específicos; }\end{array}$ & $\begin{array}{l}\text { Mínimo de uma } \\
\text { atividade/mês; }\end{array}$ & Questionário \\
\hline & Média de Visita domiciliar por família; & $\begin{array}{l}\text { Igual ou superior a uma } \\
\text { visita/mês; }\end{array}$ & Questionário \\
\hline & $\begin{array}{l}\text { População cadastrada e acompanhada por } \\
\text { grupo etário: }<5 \text { anos e > } 60 \text { anos; }\end{array}$ & $70 \%$ ou mais; & Formulário \\
\hline & $\begin{array}{l}\text { Diabéticos e hipertensos cadastrados e } \\
\text { acompanhados }\end{array}$ & $90 \%$ ou mais; & Formulário \\
\hline & Cobertura vacinal por tetra $<1$ ano & 95\%; & Formulário \\
\hline & Cobertura vacinal para influenza $>60$ anos & 75\%; & Formulário \\
\hline & Composição da ESF; & $\begin{array}{l}100 \% \text { nos últimos seis } \\
\text { meses; }\end{array}$ & Questionário \\
\hline & $\begin{array}{l}\text { Regularidade e suficiência da provisão de } \\
\text { suprimentos (insumos e medicamentos) e } \\
\text { equipamentos; }\end{array}$ & $\begin{array}{l}\text { Regularidade e } \\
\text { suficiência nos últimos } \\
\text { três meses; }\end{array}$ & Questionário \\
\hline & $\begin{array}{l}\text { Apoio diagnóstico e referência para atenção } \\
\text { especializada; }\end{array}$ & $100 \%$ & Questionário \\
\hline & $\begin{array}{l}\text { Contra-referência para o nível básico dos } \\
\text { pacientes atendidos em serviço de nível } \\
\text { secundário e terciário; }\end{array}$ & $\begin{array}{l}\text { Relatório escrito em } \\
100 \% \text {; }\end{array}$ & Questionário \\
\hline & $\begin{array}{l}\text { Acompanhamentos aos usuários no } \\
\text { domicílio, egressos de internações sensíveis à } \\
\text { atenção básica; }\end{array}$ & 100\% das internações; & Questionário \\
\hline \multirow{4}{*}{ Longitudinalidade } & Carga horária dos profissionais da ESF; & 40 horas semanais; & Questionário \\
\hline & $\begin{array}{l}\text { Médicos e enfermeiros com treinamento } \\
\text { introdutório; }\end{array}$ & $\begin{array}{l}\text { Realizado nos primeiros } \\
\text { três meses de atuação; }\end{array}$ & Questionário \\
\hline & ESF com tempo de funcionamento estável; & $\begin{array}{l}\text { Igual ou maior do que } \\
\text { um ano; }\end{array}$ & Questionário \\
\hline & $\begin{array}{l}\text { Tempo de atuação do médico e do } \\
\text { enfermeiro na ESF; }\end{array}$ & $\begin{array}{l}\text { Igual ou maior do que } \\
\text { seis meses; }\end{array}$ & Questionário \\
\hline
\end{tabular}

A categorização das duas unidades ecológicas pretendidas foi desenvolvida em três etapas, representadas na Figura 1. A primeira etapa visou identificar a cobertura populacional pelo PSF em dezembro 2004, utilizando as informações do Pacto de Indicadores da Atenção Básica 2004. 


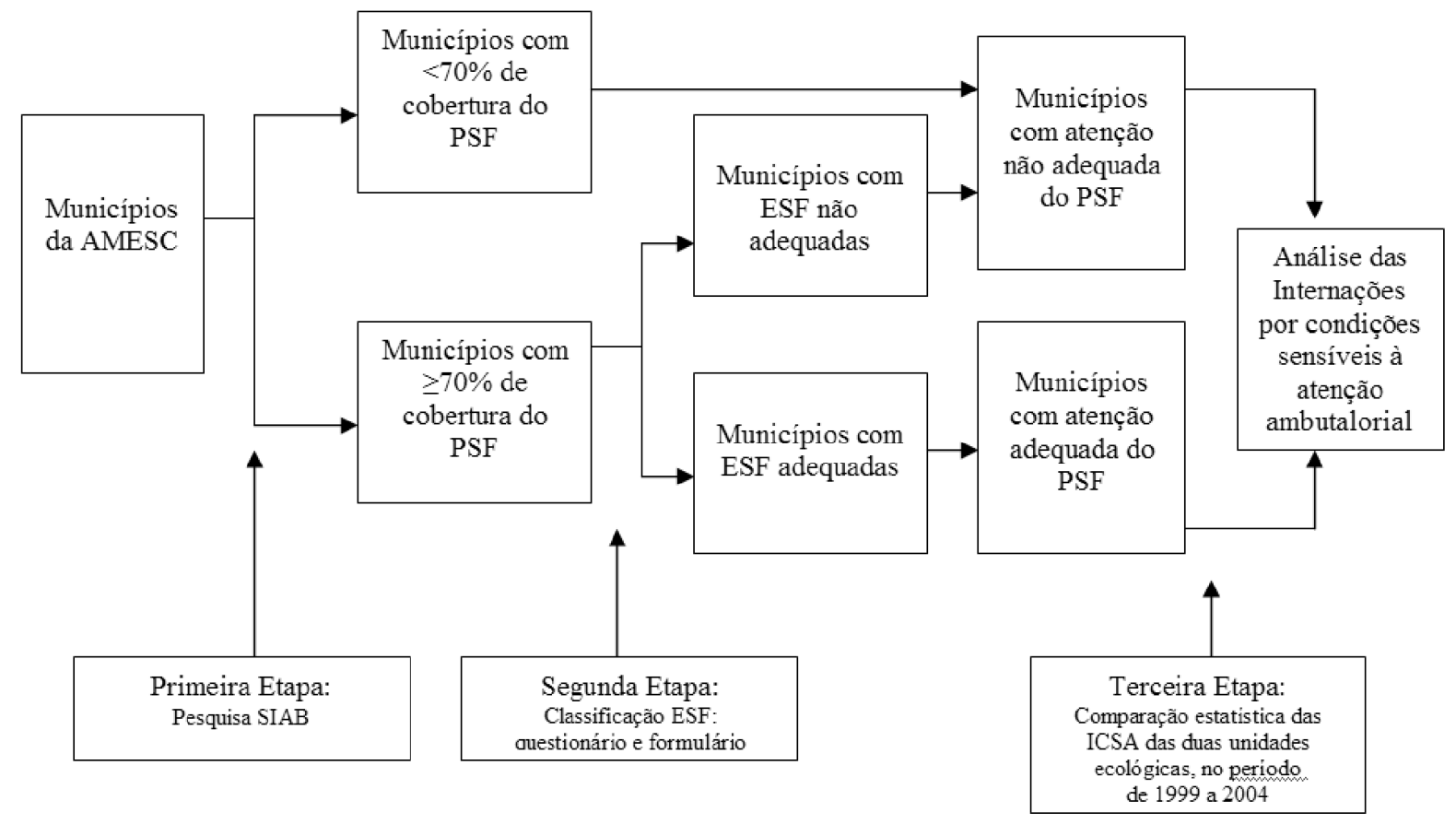

Figura 1 - Concepção da pesquisa.

Figure 1 - Research design.

Assim, os 15 municípios da AMESC foram classificados como municípios adequados ou não adequados em relação à cobertura, formando duas unidades ecológicas preliminares. Inicialmente, a primeira unidade ecológica, que incluiu os municípios com $70 \%$ ou mais de cobertura populacional do PSF, somou doze municípios, e a segunda unidade ecológica, com cobertura do PSF abaixo de $70 \%$, agregou três municípios.

Na segunda etapa, os doze municípios considerados adequados pela cobertura do PSF foram reclassificados a partir da avaliação da qualidade da atenção oferecida à população pelas ESFs instaladas. As 34 ESFs destes municípios foram estudadas, analisando-se componentes de estrutura e processo que influenciam a sua prática, agrupados segundo alguns dos padrões ordenadores da Atenção Primária à Saúde e da Estratégia da Saúde da Família - ESF assumidos como relevantes para a construção da qualidade da atenção oferecida à população ${ }^{5,10,29-34}$. (Tabela 1)

A obtenção da pontuação que definiu a adequação ou não da atenção oferecida pelas ESFs resultou da aplicação de ques- tionário, respondido por $100 \%$ das equipes, onde elementos de estrutura e processo adequados à atenção foram pontuados (total de 90 pontos) em quarenta e cinco perguntas fechadas; e do formulário preenchido pela pesquisadora, a partir de dados do Sistema de Informação da Atenção Básica - SIAB, pontuando (total de 10 pontos) outros elementos de estrutura e processo complementares para a adequada atenção à saúde nestes municípios. As equipes que pontuaram 75 pontos ou mais foram consideradas adequadas para desenvolver atenção básica de qualidade mínima.

Com os dados obtidos, os municípios foram reclassificados, ou seja, para que estes continuassem a compor a primeira unidade ecológica, que agregou os municípios com atenção básica adequada, além da cobertura igual ou maior de $70 \%$, pelo menos dois terços de suas equipes deveriam estar adequados para desenvolver atenção básica com qualidade ( 75 pontos ou mais no questionário/formulário). Dos doze municípios considerados adequados em cobertura na primeira etapa, somente nove apresentaram equipes com 75 pontos ou mais. 
Na configuração final das unidades ecológicas estudadas, considerando em conjunto a cobertura e a qualidade das equipes segundo os critérios já citados, ficou assim definido: a primeira unidade ecológica foi constituída por nove municípios da região, com população total residente, em 2004, de 124.152 habitantes. À segunda unidade ecológica, que continha três municípios considerados não adequados pela cobertura do PSF, somaram-se mais três municípios, pois suas equipes foram consideradas qualitativamente não adequadas em relação às ações de atenção básica oferecidas à população, com um total de 44.477 habitantes neste mesmo ano.

Assim, as duas unidades ecológicas de análise foram conformadas, agrupando municípios com atenção adequada do PSF (nove municípios) e municípios com atenção não adequada do PSF (seis municípios).

Na terceira etapa, a pesquisa levantou dados referentes ao comportamento evolutivo das internações por algumas causas sensíveis à atenção ambulatorial (Diabete Mellitus, Pneumonia em menores de cinco anos e maiores de sessenta anos, Diarréia em menores de cinco anos, AcidenteVascular Cerebral e Infarto Agudo do Miocárdio), assumidos como indicadores de resultado, e explorou as diferenças encontradas entre as duas unidades ecológicas no período de 1999 a 2004.

Para testar o comportamento evolutivo das ICSAAs selecionadas, neste período, foi utilizada a análise de regressão linear simples, enfatizando o coeficiente de regressão linear (tendência), o coeficiente de correlação de Pearson e sua significância. Neste estudo foi adotado o nível de significância de $5 \%$.

Para o cálculo das taxas de ICSAAs foram pesquisadas as internações por residência nos municípios com atenção básica adequada e não adequada (1999-2004), cujos diagnósticos principais segundo código da $10^{\text {a }}$ Revisão da Classificação Internacional da Doença (CID-10) eram: Diabete Mellitus (E.10, E.14); Pneumonia (J.12, J.18) em me- nores de cinco anos e maiores de sessenta anos; Diarréia (A.09) em menores de cinco anos; AcidenteVascular Cerebral - AVC (I.64) e Infarto Agudo do Miocárdio - IAM (I.21). As taxas de internações foram calculadas seguindo a metodologia da Portaria № 21/ GM de 5 de janeiro de 2005 dos Indicadores do Pacto da Atenção Básica. Os dados utilizados tiveram como origem o Sistema de Informações Hospitalares (SIH-SUS) e IBGE.

Em síntese, a atenção "adequada" à população foi comparada com a atenção "não adequada" em relação à capacidade de influenciar as taxas de internação hospitalar pelas causas sensíveis à atenção ambulatorial acima indicada. O comportamento dessas internações foi assumido como indicador de resultado da ação do PSF.

A concepção da pesquisa, conforme a descrição acima, pode ser representada de forma mais sintética pela Figura 1.

\section{Resultados}

A avaliação das internações por algumas condições sensíveis à atenção ambulatorial - ICSAAs na população residente na região da AMESC, no período de 1999 a 2004, segundo municípios com atenção básica adequada ou não adequada conforme critérios já apresentados, pode ser resumida nas Tabelas 2 e 3.

\section{Internações por Diabetes Mellitus}

Observando-se a Figura 2, percebe-se que nos municípios com atenção não adequada, as taxas de internações por este agravo não revelam tendência de decréscimo ao longo do período investigado. Enquanto que nos adequados, observa-se uma tendência linear decrescente, embora não significativa estatisticamente.

\section{Internações por pneumonia da população menor (<) de 5 anos}

A tendência de decréscimo anual destas internações (Figura 3) ocorreu tanto 
Tabela 2 - Taxas, Tendência, Correlação de Pearson e p-valor para internações por Diabete Mellitus, AVC, IAM (por 10.000 hab.), Pneumonia em $<5$ anos e $>60$ anos, diarréia $<5$ anos (por 1.000 hab.) da população residente em municípios com PSF adequado - AMESC, 1999-2004.

Table 2 - Rates, Trend, Pearson's Correlation and p-value for admissions due to Diabetes Mellitus, Stroke, MI (per 10,000 inhab.), Pneumonia in $<5 y$-o and $>60 y$-o, Diarrhea $<5 y$-o (per 1,000 inhab.) in the resident population in cities with appropriate PSF, AMESC, 1999-2004.

\begin{tabular}{lcccccc}
\hline Ano & DM/10000 & $\begin{array}{c}\text { Pneum<5 } \\
\text { anos/1000 }\end{array}$ & $\begin{array}{c}\text { Pneum }>60 \\
\text { anos/1000 }\end{array}$ & $\begin{array}{c}\text { Diar }<5 \\
\text { anos/1000 }\end{array}$ & AVC/10000 & IAM/10000 \\
\hline 1999 & 16,86 & 24,36 & 19,06 & 0,07 & 1,45 & 4 \\
2000 & 11,99 & 27,48 & 14,86 & 0,08 & 2,47 & 2,89 \\
2001 & 11,39 & 22,93 & 13,74 & 0,00 & 1,09 & 4,18 \\
2002 & 17,76 & 21,63 & 13,96 & 0,08 & 11,90 & 5,29 \\
2003 & 13,37 & 25,61 & 7,80 & 0,00 & 19,49 & 5,54 \\
2004 & 12,00 & 19,64 & 10,66 & 0,10 & 14,82 & 5,24 \\
Tendência & $-0,394$ & $-0,871$ & $-1,799$ & $-0,000$ & $+3,677$ & $+0,436$ \\
Correlação de Pearson & $-0,269$ & $-0,578$ & $-0,878$ & $-0,012$ & $+0,869$ & $+0,826$ \\
P valor & 0,606 & 0,228 & 0,021 & 0,981 & 0,024 & 0,055 \\
\hline
\end{tabular}

Fonte: SIH-SUS, IBGE.

Source: SIH/SUS, IBGE.

Tabela 3 - Taxas, Tendência, Correlação de Pearson e p-valor para internações por Diabete Mellitus, AVC, IAM (por 10.000 hab.), Pneumonia em $<5$ anos e $>60$ anos, diarréia $<5$ anos (por 1.000 hab.) na população residente em municípios com PSF não adequado - AMESC, 1999-2004.

Table 3 - Rates, Trend, Pearson's Correlation and p-value for admissions due to Diabete Mellitus, Stroke, MI (per 10,000 inhab.), Pneumonia in $60 \mathrm{y}-0$, Diarrhea, $<5 \mathrm{y}-0$ (per 1,000 inhab.) in the resident population of cities with inappropriate PSF, AMESC, 1999-2004.

\begin{tabular}{lcccccc}
\hline Ano & DM /10000 & $\begin{array}{c}\text { Pneum<5 } \\
\text { anos/1000 }\end{array}$ & $\begin{array}{c}\text { Pneum }>60 \\
\text { anos/1000 }\end{array}$ & $\begin{array}{c}\text { Diar }<5 \\
\text { anos/1000 }\end{array}$ & AVC/10000 & IAM/10000 \\
\hline 1999 & 17,76 & 19,09 & 19,38 & 0,84 & 12,07 & 1,89 \\
2000 & 23,23 & 27,73 & 16,36 & 0,97 & 21,19 & 2,82 \\
2001 & 17,60 & 21,85 & 20,98 & 0,58 & 6,02 & 2,78 \\
2002 & 17,21 & 14,25 & 13,08 & 0,95 & 21,11 & 5,05 \\
2003 & 16,13 & 17,32 & 13,19 & 0,94 & 15,22 & 5,22 \\
2004 & 22,03 & 16,60 & 14,95 & 0,75 & 11,02 & 3,82 \\
Tendência & $-0,097$ & $-1,465$ & $-1,130$ & $-0,004$ & $-0,230$ & $+0,546$ \\
Correlação de Pearson & $-0,006$ & $-0,573$ & $-0,647$ & $-0,006$ & $-0,072$ & $+0,762$ \\
P valor & 0,990 & 0,233 & 0,164 & 0,910 & 0,892 & 0,077 \\
\hline
\end{tabular}

Fonte: SIH-SUS, IBGE.

Source: SIH/SUS, IBGE.

na unidade ecológica atendida de forma adequada quanto naquela que recebeu atenção considerada não adequada, embora o coeficiente de tendência não se apresentasse estatisticamente significativo nas duas unidades.
Internações por pneumonia da população maior (>) de 60 anos

A avaliação da taxa de internação em relação a esta causa (Figura 4) sugere tendência de redução das internações por 


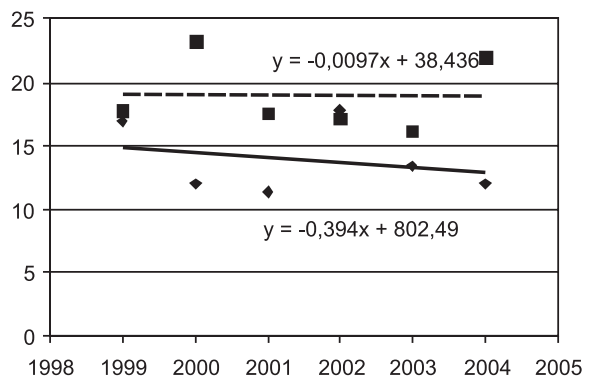

- Adequado

- Não Adequado

- Linear (Adequado)

- - Linear (Não Adequado

Figura 2 - Distribuição das taxas de internações por Diabete Mellitus (por 10.000 hab.), nos municípios com PSF adequado e não adequado, AMESC, 1999-2004.

Fonte: SIH-SUS, IBGE.

Figure 2 - Distribution of admission rates for Diabete Mellitus (per 10,000 inhab.), in cities with appropriate and inappropriate PSF, AMESC, 1999-2004.

Source: SIH/SUS, IBGE.

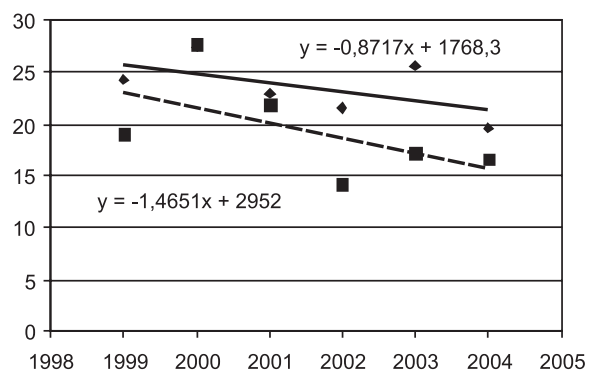

- Adequado

- Não Adequado

- Linear (Não Adequado)

- Linear (Adequado)

Figura 3 - Distribuição das taxas de internações por pneumonia $<5$ anos (por 1.000 hab.),

nos municípios com PSF adequado e não adequado, AMESC, 1999-2004.

Fonte: SIH-SUS, IBGE.

Figure 3 - Distribution of admission rates for pneumonia in < 5 y-o (per 1,000 inhab.), in cities with appropriate and inappropriate PSF, AMESC, 1999-2004.

Source: SIH/SUS, IBGE.

pneumonia nas duas unidades ecológicas, no período de 1999 a 2004. Nos municípios com atenção adequada, o decréscimo por unidade de tempo é maior do que nos não adequados. Nos municípios com atenção adequada, o coeficiente de tendência decresceu com taxas maiores.

Estatisticamente, podemos dizer que existe uma forte correlação entre as variáveis estudadas, sugerindo associação entre as ações adequadas das ESFs e o declínio das internações por pneumonia na população > 60 anos residente nestes municípios.

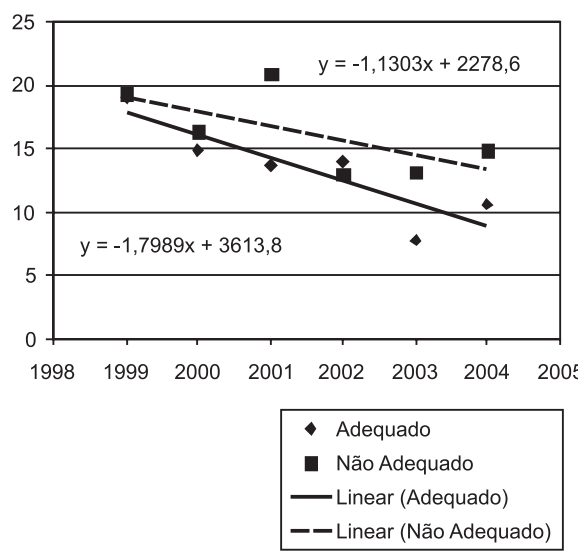

Figura 4 - Distribuição das taxas de internações por pneumonia na população > 60 anos (por 1.000 hab.) nos municípios que apresentam PSF adequado e não adequado, AMESC, 1999-2004.

Fonte: SIH/SUS, IBGE.

Figure 4 - Distribution of admission rates for pneumonia in the $>60 y$-o population (per 1,000 inhab.) in the cities with appropriate and inappropriate PSF, AMESC, 1999-2004. Source: SIH/SUS, IBGE.

\section{Internações por diarréia na população menor (<) de 5 anos}

O estudo evidenciou mudanças pouco significativas nestas taxas de internação, nas duas unidades ecológicas. Embora a análise estatística não revele tendência significativa, a taxa de crescimento médio por 1.000 habitantes, nos municípios com atenção adequada foi de 0,05 casos nos seis anos estudados, enquanto que naqueles com atenção não adequada, no mesmo período, a taxa de crescimento médio foi de 0,84 por 1.000 habitantes. 


\section{Internações por Acidente Vascular Cerebral (AVC)}

A análise estatística (Figura 5) mostrou tendência significativa e correlação entre as variáveis estudadas, com tendência ao aumento das internações por AVC na população atendida pelo PSF adequado, demonstrando associação entre as variáveis estudadas. Nos municípios com PSF não adequado, demonstrou uma associação fraca e tendência tênue, não significativa, de queda linear entre as variáveis estudadas.

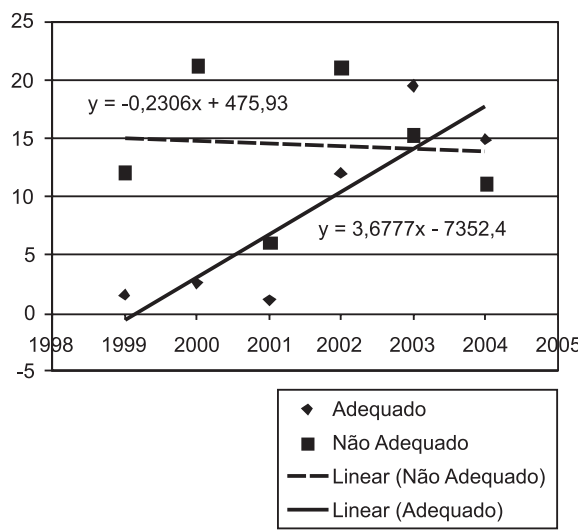

Figura 5 - Distribuição das taxas de internações por AVC (por 10.000 hab.), nos municípios com PSF adequado e não adequado, AMESC, 1999-2004.

Fonte: SIH/SUS, IBGE.

Figure 5 - Distribution of admission rates for stroke (per 10,000 inhab.), in cities with appropriate and inappropriate PSF, AMESC, 1999-2004.

Source: SIH/SUS, IBGE.

\section{Internações por Infarto Agudo do Miocárdio (IAM)}

Na Figura 6 podemos verificar o comportamento ascendente das taxas de internação por Infarto Agudo do Miocárdio tanto da população dos municípios com atenção não adequada quanto da população dos municípios com atenção adequada na atenção básica. As duas unidades ecológicas demonstraram tendência linear crescente positiva, estatisticamente significativa em nível de significância menor que $10 \%$. A tendência nos municípios com atenção adequada foi menor do que naqueles com atenção não adequada.

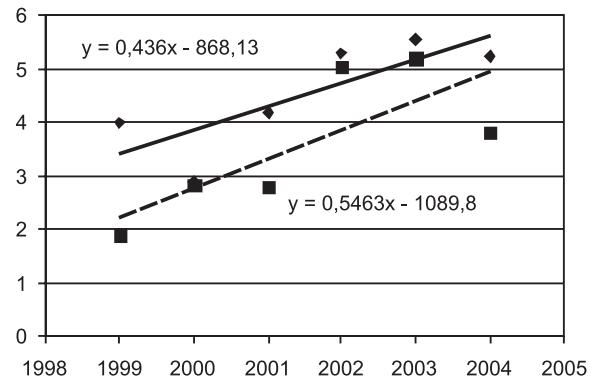

$$
\begin{aligned}
& \text { Adequado } \\
& \text { - Não Adequado } \\
& \text { - Linear (Não Adequado) } \\
& \text { — Linear (Adequado) }
\end{aligned}
$$

Figura 6 - Distribuição das taxas de internações por IAM (por 10.000 hab.) nos municípios com PSF adequado e não adequado, AMESC, 1999-2004.

Fonte: SIH/SUS, IBGE.

Figure 6 - Distribution of admission rates for $\mathrm{MI}$ (per 10,000 inhab.) in cities with appropriate and inappropriate PSF, AMESC, 1999-2004. Source: SIH/SUS, IBGE.

\section{Discussão}

Os resultados recém apresentados evidenciaram mudanças nas taxas de internação de todas as condições mórbidas analisadas, apontando tendência de aumento e/ou declínio dessas internações no período estudado nas duas unidades ecológicas da pesquisa. A discussão sobre os fatores que influenciaram os resultados observados no conjunto das internações estudadas, especialmente aquelas associadas à estrutura, aos processos e à qualidade dos serviços oferecidos pelas ESF, é fundamental para a análise proposta neste trabalho.

A estratégia metodológica adotada pela pesquisa, qualificando os serviços antes de medir seus resultados, permitiu identificar fragilidades na institucionalização e funcionamento do sistema de saúde, que, em geral, conduzem à violação de alguns princípios da APS, tal como definidos por 
Starfield ${ }^{5}$, comprometendo os objetivos propostos para esse nível de atenção.

Neste sentido, os problemas associados à baixa cobertura nas faixas menores de cinco anos, a deficiência nas visitas domiciliares, a falta das atividades dirigidas à população adscrita, à manutenção do atendimento por demanda espontânea e a outros obstáculos administrativos ou técnicos, acabam por dificultar o acesso às unidades de saúde da família, deixando os hospitais como a única porta de entrada para o sistema de saúde ${ }^{34}$.

Os resultados apresentados, a par de comprovar o avanço ocorrido na última década, constatam o caráter precário das mudanças ocorridas desde o início da implementação do PSF, especialmente quando se constata a indefinição das políticas relacionadas com o processo de trabalho e a insuficiente qualificação das equipes de saúde da família. Confirmando esta afirmação, na região da AMESC apenas $14 \%$ das equipes consideradas adequadas receberam capacitação através de Curso Introdutório, menos da metade dos médicos das equipes de PSF (45\%) cumprem a jornada de 40 horas semanais e mais de $25 \%$ das equipes se modificam em tempo menor do que doze meses. Há evidências de que a qualificação do profissional de saúde é um dos desafios para que se alcance maior qualidade dos serviços de atenção à saúde. A formulação de contratos de trabalho que garantam maior estabilidade e fortaleçam o vínculo do profissional com o serviço e a população atendida são desafios necessários e indissociáveis ao sucesso do PSF como estratégia de reorientação do modelo de atenção à saúde no Brasil ${ }^{35-37}$.

A realidade observada conflita com as propostas teóricas associadas à implementação do PSF. A assistência qualificada à saúde da população exige continuidade na atenção básica, além da eliminação de fatores organizacionais diversos que dificultam a adesão dos clientes e impedem o efetivo controle dos agravos não infecciosos $^{7}$. Este estudo demonstrou as inúmeras dificuldades enfrentadas pelos profissionais das equipes de saúde da família para a realização de seu trabalho, dificuldades estas que produzem impacto sobre o resultado da assistência quando mensurada pela ótica das internações por causas sensíveis à atenção ambulatorial.

Assim, quando os suprimentos nem sempre estão disponíveis com a regularidade desejada, as deficiências dos sistemas de apoio diagnóstico são obstáculos à boa prática clínica, tais como as dificuldades para manter sob controle os níveis glicêmicos e a hipertensão arterial, a falta de equipamentos e medicamentos essenciais é uma constante, os obstáculos encontrados para a referência e contra referência aos serviços dos níveis secundários e terciários são relevantes, o resultado é o aumento de patologias que por falta de diagnóstico e controle oportunos, agravam e têm sua atenção adequada apenas no nível hospitalar.

Assim, podemos avaliar a atenção básica realizada por meio do PSF na região da AMESC como parcialmente efetiva para controle dos agravos selecionados para o estudo. Essa eficácia parcial pode ser resultado da explicitação de uma demanda até então latente e que o próprio PSF se encarregou de explicitar e encaminhar para atenção adequada. É sabido que, ao se ampliar o acesso aos serviços de saúde, a demanda por estes também se eleva, aumentando a ocorrência de internações. Outro aspecto pouco estudado refere-se à possibilidade de que alguns agravos tenham sensibilidade reduzida à atenção adequada, ou seja, alguns problemas de saúde podem estar sendo bem atendidos, mas terão seus indicadores de resultado modificados longo tempo depois de iniciada essa atenção.

Finalmente, a determinação e o condicionamento social de vários problemas de saúde avaliados no decorrer deste estudo indicam que a disponibilidade de outros recursos associados ou não à prática médica, especialmente os associados às condições socioeconômicas dos grupos sociais sob estudo, pode definir o resultado de forma mais direta do que considerando aspectos da atenção à saúde recebida pelos mesmos. 
Estudos realizados por Caminal et al. ${ }^{17} \mathrm{e}$ Komaromy et al. ${ }^{19}$, demonstraram que significativa proporção de internações evitáveis é ainda dependente da disponibilidade de recursos e da prática médica.

Ao mesmo tempo, uma maior oferta de leitos hospitalares por habitante pode significar, muitas vezes, recursos excedentes e ociosos. Diante desta possibilidade de recursos, os resultados sugerem uma maior predisposição médica em internar. Por outro lado, segundo ainda os autores, esse resultado pode sugerir que uma oferta excedente na atenção hospitalar pode induzir o município a direcionar menos esforços para a gestão da APS, provocando o agravo de doenças que poderiam ser tratadas precocemente.

No sentido inverso, mas com resultados muito parecidos, quando o saber corrente indica que o conhecimento da provisão da APS é inadequado na região, este pode estimular o médico a efetuar a internação precoce como alternativa de tratamento, uma vez que o cuidado mínimo para o caso poderia ficar comprometido com a atenção ambulatorial disponível.

Como contribuição para uma explicação mais consistente com os resultados obtidos, que dependem de outros níveis de atenção para avaliação da atenção primária, está a possibilidade de que a maior oferta de leitos hospitalares $^{9}$ da região estudada, localizada exatamente nos municípios que apresentam atenção básica mais qualificada, possa influenciar os padrões de internação da população desses municípios. A favor desta hipótese podemos incluir o fato de que, das sete unidades hospitalares existentes na região, cinco unidades estão localizadas nos municípios com atenção básica oferecida por PSF considerado adequado.

Além disso, o padrão de morbidade, o comportamento de procura por cuidado à saúde e o estilo de vida dos pacientes são outros fatores que podem influir sobre este conjunto de hospitalizações. Dependendo da predisposição clínica e social do médico em hospitalizar ou não o paciente, a taxa de internação evitável pode ser afetada ${ }^{9,17}$. A propensão social do médico para hospitalizar pode ser maior em áreas mais pobres, uma vez que nestes locais os pacientes são mais vulneráveis a complicações médicas e a fatores ambientais ${ }^{19}$.

Relativizando os efeitos da atenção do PSF sobre algumas doenças, como o Diabete Mellitus e a Hipertensão Arterial, que dependem do cuidado à saúde ao longo de um período de tempo relativamente longo, constatamos que a implantação do PSF nos municípios estudados é relativamente recente, definindo possibilidades limitadas quanto ao impacto sobre estas doenças.

Assim, tendo em vista as determinações e os condicionantes diferenciados que interferem na linha de cuidado de cada patologia estudada e na própria história natural de cada uma dessas patologias, a atuação da ESF sobre as mesmas pode ter diferentes tempos de carência para efetivar alterações estatísticas captadas em estudos como o aqui realizado. Os efeitos desta atenção sobre algumas doenças como Hipertensão e Diabete Mellitus, pode depender de um tempo de carência maior que o da implantação do PSF na região da AMESC. Transformar hábitos e condutas - parte integrante do processo de transformação das patologias com forte componente comportamental, exige mais do que isso - o sucesso da equipe depende da adesão dos pacientes, pois a incorporação de novos hábitos e estilos de vida demanda tempo para ser internalizada e apreendida ${ }^{7}$.

Mesmo com a possível interferência desses fatores, pode-se perceber no conjunto dos resultados avaliados através desse estudo que, mesmo limitadas ao espaço da região estudada - a AMESC, ocorreram mudanças positivas na evolução de algumas taxas de internações por condições sensíveis à atenção ambulatorial, podendo estar associadas a priori à oferta de atenção básica através do PSF de qualidade. A magnitude das mudanças, em geral mais significativa nos municípios onde a oferta da atenção básica do PSF é mais adequada, sugere que a ampliação da cobertura do PSF facilitou o acesso da população aos serviços deste 
nível de atenção, e que ao mesmo tempo a qualificação da atenção recebida contribuiu para a redução das internações pelas causas selecionadas.

\section{Considerações finais}

Ao se adotar o PSF como estratégia estruturante da reorientação do modelo de atenção à saúde na região da AMESC, a avaliação desse processo configurou-se como uma proposta pertinente na medida em que os efeitos positivos sobre a saúde da população são perceptíveis tanto de forma objetiva quanto subjetiva. Ao mesmo tempo, é cada vez mais visível o impacto do Sistema Único de Saúde na situação de saúde de uma dada população, seja pelo papel relevante desempenhado pelos serviços de atenção primária no cotidiano dos serviços, seja pela redução da busca por serviços de atenção se- cundários e terciários, conforme resultados apresentados no presente trabalho.

É sob esta perspectiva que as equipes de saúde vêm desenvolvendo um enorme esforço buscando a sistematização de saberes e práticas adaptados para o espaço local e para o novo modelo de atenção, mostrando que a produção do conhecimento pode ser vivenciada e construída no espaço dos serviços, mesmo com as dificuldades do cotidiano encontradas nos elementos de estrutura $\mathrm{e}$ processo que permeiam as suas ações. Os dados de desempenho da atenção primária avaliados neste estudo revelam dificuldades, mas também inovações e uma perspectiva de transformação concreta da prática médicosanitária tradicional. O certo é que esse processo é movido pelos desafios que se colocam todos os dias para os trabalhadores de saúde, mobilizando corações e mentes para a conquista da saúde para toda a população.

\section{Referências}

1. Brasil. Projeto promoção da saúde: as cartas da promoção da saúde. Brasília, DF: Secretaria de Políticas de Saúde; 2002.

2. Aleixo JLM. Atenção Primária à Saúde e o Programa de Saúde da Família: perspectiva de desenvolvimento no início do terceiro milênio. Rev Mineira Saúde Pública 2002; 1(1): 1-16.

3. Almeida C. Novos modelos de atenção à saúde: bases conceituais e experiências de mudança. In: NR Costa \& JM. Ribeiro (org.). Política de saúde e inovação institucional: uma agenda para os anos 90. Rio de Janeiro: Escola Nacional de Saúde Pública, Fiocruz; 1996.

4. Brasil. Coordenação de Saúde da Comunidade, Secretaria de Assistência à Saúde. Saúde da Família: uma estratégia para a reorientação do modelo assistencial. Brasília; 1997.

5. Starfield B. Atenção primária: equilíbrio entre necessidades de saúde, serviços e tecnologia. Brasília: UNESCO, Ministério da Saúde; 2002.

6. Brasil. Atenção primária. Seminário do CONASS para construção de consensos. (CONASS Documenta; v. 2). Brasília: CONASS; 2004.

7. Brasil. Conselho Nacional de Secretários de Saúde. Acompanhamento e avaliação da atenção primária. (CONASS Documenta; v. 7) Brasília; 2004.
8. Brasil. Ministério da Saúde. Secretaria de Atenção à Saúde. Departamento de Atenção Básica (Série C. Projetos, Programas e Relatórios). Monitoramento na atenção básica de saúde: roteiros para reflexão e ação. Brasília; 2004.

9. Mendes EV. A atenção primária à saúde no SUS Fortaleza. Fortaleza: Escola de Saúde Pública do Ceará; 2002.

10. Brasil. Conselho Nacional de Secretários de Saúde - CONASS. Atenção Primária e Promoção da saúde. Brasília; 2007.

11. Almeida $\mathrm{C}$ et al. Projeto: desenvolvimento de metodologia de avaliação do desempenho do sistema de saúde brasileiro (PRO-ADESS). Relatório Final. Rio de Janeiro; 2003.

12. Caminal J, Zurro AM. Sobre la contribuición de la atención primaria a la capacidad resolutiva del sistema de salud y su medición. Aten Primaria 2005; 36(6): 456 61 .

13. Perpétuo IHO, Wong LR. Atenção hospitalar por Condições Sensíveis à Atenção Ambulatorial (CSAA) e as mudanças no seu padrão etário: uma análise exploratória dos dados de Minas Gerais. Diamantina: XII Seminário sobre Economia Mineira; 2006.

14. Perrin JM. Variations in pediatric hospitalization rates: why do they occur? Pediatric Annals 1994; 23(12): 676681. [PubMed indexed for Medline]. 
15. Pappas G., Hadden WC, Kozak LJ, Fisher GF. Potentially avoidable hospitalization: Inequalities in rates between US socioeconomic groups. Am J Public Health 1997; 87(5): 811-6.

16. Laditka, J. N., Laditka, S. B., Probst, J. C. More may be better: Evidence of a negative relationship between physician supply and hospitalization for ambulatory care sensitive conditions. Health Serv Res 2005; 40(4): 1148-66.

17. Komaromy M, Lurie N, Osmond D, Vranizan K, Keane D, Bindman A. Physician practice style and rates of hospitalization for Chronic Medical Conditions. Med Care 1996; 34(6); 594-609.

18. Bermúdez-Tamayo C, Márquez-Calderón S, Águila MMR, López EPM, Espinosa O. Características organizativas de la atención primaria y hospitalización por los principales ambulatory care sensitive conditions. Aten Primária 2004; 33(6): 305-11.

19. Caminal JH, Morales M, Sánchez E, Cubells MJ, Bustins M. Hospitalizaciones prevenibles mediante una atención primaria oportuna y efectiva. Aten Primária 2003; 31(1): 6-17.

20. Calderón SM, Rodríguez MM, Milla EP, Ortiz J, Tamayo CB. Factores asociados a la hospitalización por procesos sensibles a cuidados ambulatorios en los municipios. Gac Sanit 2003; (17)5: 360-7.

21. Caminal J, Mundet X, Ponsà JA, Sánchez E, Casanova C. Las hospitalizaciones por ambulatory care sensitive conditions: selección del listado de códigos de diagnóstico válidos para Espana. Gac Sanit 2001; 15(2): 128-41.

22. Brasil. Ministério da Saúde. Secretaria de Atenção à Saúde. Departamento de Atenção Básica (Série C. Projetos, Programas e Relatórios). Saúde da família no Brasil: uma análise de indicadores selecionados: 19982004. Brasília; 2006.

23. Machado ENM, Fortes FBCTP, Somarriba M. Efeitos da introdução do PAB sobre a distribuição de recursos e a prestação de serviços: o caso de Minas Gerais. Ciência Saúde Coletiva 2004; 9(1): 99-111.

24. Silva AAM, Gomes UA, Tonial SR, Silva RA. Fatores de risco para hospitalização de crianças de um a quatro anos em São Luís, Maranhão, Brasil. Cad Saúde Pública 1999; 15(4): 749-57.

25. Peixoto HCG, Souza ML, Freitas SFT. Ordenação dos principais grupos de diagnósticos de internação no sistema único de saúde do Brasil, 1997, s/diferentes critérios. Disponível em: <www.saude.sc.gov.br/ gestores/sala_de_leitura/artigos/internações_ hospitalares $>$. [Acessado em 24 maio de 2005].

26. Santa Catarina. Projeto de fortalecimento e apoio ao desenvolvimento institucional da gestão estadual do SUS. Ministério da Saúde/REFORSUS. Fundação de Desenvolvimento da Pesquisa - FUNDEP/UFMG. Diagnóstico de saúde do Estado de Santa Catarina. Belo Horizonte, MG; 2001.
27. Santa Catarina. Secretaria de Estado do Planejamento. Programa das Nações Unidas para o Desenvolvimento - PNUD/SC. Projeto Meu Lugar. Indicadores de desenvolvimento social. SDR de Araranguá Florianópolis, SC; 2005.

28. Cadastro Nacional de Estabelecimentos de Saúde. http://cnes.datasus.gov.br/Mod_Ind_Unidade. asp?VEstado=42\&VMun=421770. [Acessado em 20 de março de 2004].

29. Donabedian A. La capacidade de la atención médica: definición e métodos de evaluacón. México: La prensa Médica Mexicana; 1984.

30. Brasil. Ministério da Saúde. Secretaria de Assistência à Saúde. Manual para a organização da atenção básica. Brasília; 1999.

31. Brasil. Ministério da Saúde. Guia prático do Programa Saúde da Família. Brasília; 2001.

32. Brasil. Ministério da Saúde. Secretaria de Atenção à Saúde. Departamento de Atenção Básica. Manual de estrutura física das unidades básicas de saúde: saúde da família. Brasília; 2006.

33. Brasil. Secretaria de Atenção à Saúde/Departamento de Atenção Básica/Coordenação de Acompanhamento e Avaliação. Documento final da comissão de avaliação da Atenção Básica. [Produto de trabalho da comissão instituída pela portaria $n^{\circ} 676 \mathrm{GM} / \mathrm{MS}$, de 3 de junho de 2003, publicadas no DOU em 4 de junho de 2003]; 2003.

34. Brasil. Ministério da Saúde/Secretaria de Assistência à Saúde, Coordenação de Atenção Básica. Avaliação da implantação e funcionamento do programa da saúde da família. Brasília; 2000.

35. Organização Pan-Americana da Saúde. Avaliação de tendências e prioridades sobre recursos humanos de saúde. Brasília: OPAS; 2002.

36. Cotta RMM, Pereira RJ, Maia TM, Marques ES, Franceschini SCC. Aprehensión y conocimiento de las directrices del Sistema Único de Salud (SUS): un reto en la consolidación de la política de salud brasileña. Rev Agathos - Atención Sociosanitaria y Bienestar 2004; 3: 16-23.

37. Organização Pan-Americana da Saúde. Informe sobre a reunião de trabalho Discussão sobre o marco referencial e conceitual da Atenção Básica e Saúde da Família no Brasil. Projeto de Desenvolvimento de Sistemas e Serviços de Saúde. Brasília: OPAS; 2003.

38. Ribeiro EM, Pires D, Blank VLG. A Teorização sobre processo de trabalho em saúde como instrumental para análise do trabalho no Programa Saúde da Família. Cad Saúde Pública 2004; 2: 438-46.

Recebido em: 22/01/08 Versão final reapresentada em: 06/07/08 Aprovado em: 10/09/08 